\title{
Phytoprotection
}

\section{Index des auteurs, volume 89 Author Index, Volume 89}

Volume 89, numéro 2-3, décembre 2008

Journée commémorative du centenaire de la société de protection des plantes du Québec

Centennial Commemoration of the Québec Society for the Protection of Plants

URI : https://id.erudit.org/iderudit/038302ar

DOI : https://doi.org/10.7202/038302ar

Aller au sommaire du numéro

Éditeur(s)

Société de protection des plantes du Québec (SPPQ)

ISSN

0031-9511 (imprimé)

1710-1603 (numérique)

Découvrir la revue

Citer cet article

(2008). Index des auteurs, volume 89. Phytoprotection, 89(2-3), 207-208.

https://doi.org/10.7202/038302ar d'utilisation que vous pouvez consulter en ligne.

https://apropos.erudit.org/fr/usagers/politique-dutilisation/ 
Index des auteurs, volume 89 Author Index, Volume 89

A-B

Akkaya, M.S.

Alder, C.

Al-Mughrabi, K.I.

Antoun, $\mathrm{H}$.

Aoun, $\mathrm{M}$.

Arsenault-Labrecque, G.

Arul, J.

Aubry, $\mathrm{O}$.

Avis, T.J.

Bacon, R.

Bala, K.

Banville, G.

Barasubiye, T.

Barrette, M.

Bauce, É.

Beaulieu, C.

Bélair, G.

Bélanger, L.

Bélanger, R.R.

Bélanger, $\mathrm{S}$.

Béliveau, C.

Bellerose, S.

Benoit, D.L.

Bernier, D.

Bernier, L.

Berthiaume, R.

Bertrand, A.

Bérubé, M.-È.

Bidhendi, G.N.

Bilodeau, É.

Boisclair, J.

Boivin, G.

Boucher, J.

Boivin, G.

Bourgeois, G.

Bourget, N.

Bouvet, G.F.

Brière, M.-H.

Brière, S.C.

Brisson, J.D.

Brodeur, J.

Brouillard, M.

Brousseau, P.-M.

\section{C-E}

Cadieux, M.

Çallak, A.

Carisse, $\mathrm{O}$.

Carrière, $\mathrm{Y}$.

Castonguay, Y.

Chagnon, M.

Charbonneau, C.

Charloteaux, B.
Charpentier, G.

186, 189

Choo, T.-M.

Chouinard, G.

Ciotala, M.

Cloutier, C.

Cloutier, J.

Cormier, D.

Côté, M.

Côté, R.

Couture, J.-N.

Couture, L.

Cusson, M.

Dallaire, $F$.

Dauphinais, N.

De Almeida, J.

De Cock, A.W.A.M.

de Ladurantaye, S.

de Ladurantaye, Y.

Delvas, N.

Dere, $\mathrm{S}$.

Despland, E.

Dewavrin, $\mathrm{F}$.

Desrosiers, N.

Dion, Y.

Dionne, A.

Dionne, J.

Domaine, É.

Domon, G.

Dostaler, D.

Doyon, J.

Dufour, J.

Dupont, A.

Eikemo, $\mathrm{H}$.

Émond, G.

Estevez, B.

Estey, R.H.

Etienne, M.

Etilé, E.

205

$179,182,198,200,200$

204

79, 183, 185, 191, 199, 202

182

179, 186, 188, 198, 200, 203, 205

205

186

204

$119,182,201$

$181,186,202,204$

186

180

186

196

187

187

187

31

197

193

185

183

187,192

182,203

188

202

$45,133,143,167$

176

191

188

201

$49,103,125,169$

192, 193

51

205

188

\section{F-K}

Fortier, A.-M.

184,185

Fournier, C.

188

Fournier, $\mathrm{F}$.

Fournier, V.

Fournier, Y.

183

185

Francis, $\mathrm{F}$.

Frève, A.

Gadoury, D.M.

Galka, B.

Garneau, A.

Gaudreau, C.

Gilbert, G.

Giraldeau, L.-A.

Girard, M.

Gök, G.

Gravel, V.
190, 195, 197

180

204

189

201

203

189

189, 190, 194

180,190

180, 206

190

31

$47,113,131$ 
Grégoire, C.

Guay, J.-F.

Hamelin, R.

Harding, C.

Haubruge, É.

Hébert, C.

Hogue, R.

Howard, R.J.

Hsiang, $T$.

Ibarzabal, J.

Jacobi, V.

Jacobs, J.

Jean, C.

Jinek, A.

Khelifi, M.

Koné, S.B.

191
191
176
7
204
$181,184,185,188,194,199$
169
180
182
184
179,191
191
79
192
187
187,192

\section{L-P}

Laberge, S.

Labrie, G.

Lachapelle, M.

Laflamme, G.

Lajeunesse, J.

Lalonde, $\mathrm{O}$.

LaRue, B.

Lasnier, J.

Laurent, F.

Leblanc, M.

Légaré, J.-P.

Legault, G.

Légère, $A$.

Lemaire, É.

Lerat, $\mathrm{S}$.

Leroux, G.D.

Lestra, M.

Letendre, $\mathrm{M}$.

Lévesque, C.A.

Long, B.

Longchamps, L.

Longpré, $\mathrm{F}$.

Lucas, É.

Maisonhaute, J.-É.

$179,186,188,192,193,197,198,202,203$

Marel, M.

Martinez, C.

Maufette, Y.

McClure, $\mathrm{M}$.

Mecteau, M.

Mecteau, M.R.

Mehrdadi, N.

Michaud, D.

Moffat, C.

Morisset, $\mathrm{O}$.

Néron, R.

Nisole, A.

Nguyen, N.

Nguyen, T.T.A.

Norvez, O.

Olivier, C.

Outreman, $\mathrm{Y}$.

Pageau, D.

Panneton, B.

Pelletier, F.

Peres-Neto, $P$.

Perron, J.-M.
Peters, R.D. $\quad 180$

Philion, V. 201

Pichette, A. 200

Pouleur, S. 201

Q-Z

Quezada-Garcia, R. 201

Rasoolizadeh, A. 202

Régnière, J. 177

Rémus-Borel, W. 191

Rhéaume, A.-J. 202

Rioux, D. 115, 179, 192

Rioux, S. 143, 183

Robideau, G.P. 196

Roullé, N. 202

Roy, M. 176, 194, 195, 197, 204

Ruel, J.-C.

194

Saguez, J.

St-Onge, M.

Santini, S.

Savard, M. 205

Savard, M.E. 200

Seifert, K.A. $\quad 180$

Shinners-Carnelley, T. 180

Shirdam, R. 21

Sholberg, P. $\quad 201$

Silk, P.J. 181

Simard, L. $\quad 37,203,204$

Simard, M. 115, 192

Simard, M.-J. $\quad 196$

Simon, J.-C. 183

Sparry, E. 205

Stensvand, A. 201

Stevenson, F.C.

Stewart, D. $\quad 202$

Sweeney, J. 181

Tartier, L.-M. 49, 77, 113, 165

Thompson, O. 180

Todorova, S. 198, 203

Toussaint, V. 204

Tremblay, G. $\quad 183$

Turgeon, J. 183

Turkington, T.K. 201

Tweddell, R.J. 1, 113, 187, 192

Vanasse, A. 183, 194

Vandermoten, S. 204

van Herk, W.G

Vanoosthuyse, F. 205

Varady-Szabo, H. 205

Vernon, R.S.

Vigier, B. 205

Villeneuve, C. 183

Vincent, C. 203

Voynaud, L. 193

Watson, A.K. 192

Work, T.T. 191, 196, 206

Wu, G.-M. 206

Zand, A.D. $\quad 21$

Zeybek, A. 31

Zoghlami, S. 200 